\title{
Recherche sur la surveillance et la prévention du suicide en Nouvelle-Écosse : pertinence de l'utilisation des dossiers des médecins légistes
}

\author{
L. A. Campbell, M. Sc. (1, 2, 7); L. Jackson, Ph. D. (3, 4); R. Bassett, Ph. D. (5); M. J. Bowes, M.D. (6); \\ M. Donahue, M. Th., M.R.S.S.A. (en cours) (7); J. Cartwright, M.A. (2); S. Kisely, M.D., Ph. D. (1,2,8)
}

Cet article a fait l'objet d'une évaluation par les pairs.

\section{Résumé}

Introduction : Les dossiers des médecins légistes peuvent nous aider à mieux comprendre l'ampleur du suicide dans une population, ainsi que les facteurs sociodémographiques ou autres associés au phénomène.

Méthodologie : Cette étude pilote avait pour objectif principal de déterminer, à l'aide de méthodes mixtes, les sources et les types de renseignements contenus dans les dossiers du Service du médecin légiste de la Nouvelle-Écosse (SMLNE) susceptibles d'orienter la surveillance du suicide et de cibler les efforts de prévention. L'objectif secondaire était de dresser un portrait de la cohorte d'une année composée de 108 personnes décédées par suicide en 2006, à la lumière des renseignements sociodémographiques accessibles et de l’utilisation des soins de santé dans l'année précédant le décès.

Résultats : L’extraction des données a révélé des incohérences à la fois dans les dossiers et d'un dossier à l'autre quant au type et à la quantité de données sociodémographiques ou autres recueillies, empêchant ainsi toute analyse de corrélation. Toutefois, le recoupement des dossiers et des bases de données a révélé une utilisation fréquente des services de santé au cours du mois précédant le décès.

Conclusion : L'introduction d'une collecte systématique de données lors des enquêtes du SMLNE permettrait de produire un ensemble de données exhaustives qui seraient utiles pour l'élaboration de politiques et la recherche à l'échelle de la population.

Mots-clés : suicide, surveillance en population, médecin légiste, coroner, données administratives

\section{Introduction}

Chaque année, quelque 90 décès par suicide sont enregistrés en Nouvelle-Écosse. Le suicide constitue donc un énorme problème de santé publique, quoiqu'il soit en grande partie évitable ${ }^{1}$. En plus d'être une expérience très traumatisante pour les membres de la famille et les amis, le suicide a un coût élevé. Le nombre d'années potentielles de vie perdues (APVP) attribuables au suicide est considérable : chez les gens de moins de 74 ans, seuls les cancers (tous les types), les maladies $\mathrm{du}$ système circulatoire et les blessures non intentionnelles ont été à l'origine d'un nombre plus élevé d'APVP de 2005 à $2007^{2}$. Comme on estime généralement que les cas de suicide ne sont pas toujours déclarés, il est possible que ces chiffres soient sous-évalués.

De nombreux facteurs contribuent à la sous-déclaration des cas de suicide, comme le fait de n'avoir pu soupçonner un suicide (en particulier chez les gens âgés ou en l'absence de message ou de toute autre indication d'un suicide possible). De plus, il est parfois particulièrement difficile de déterminer l'intention, dans le cas d'une intoxication par exemple. Le taux de suicide par intoxication pourrait être sous-estimé d'environ $30 \%$, alors que la sous-déclaration est de $10 \%$ dans le cas du taux global de suicide ${ }^{3}$. Une étude ontarienne sur la validité des actes de décès d'adultes décédés d'une cause non naturelle a fait ressortir la difficulté de déterminer l'intention en raison de la subjectivité de l'interprétation ${ }^{4}$. Les décès par pendaison ou par inhalation d'un gaz nocif étaient plus susceptibles d'être attribués à un suicide que les décès par intoxication ou par noyade; la mort par surdose de médicaments en vente libre était plus souvent attestée comme étant un suicide que le résultat d'une surdose d'héroïne. Une preuve plus solide de l'intention augmentait les chances de classer correctement le

Rattachement des auteurs :

1. Département de psychiatrie, Université Dalhousie, Halifax (Nouvelle-Écosse), Canada

2. Département de santé communautaire et d'épidémiologie, Université Dalhousie, Halifax (Nouvelle-Écosse), Canada

3. École de la santé et de la performance humaine, Université Dalhousie, Halifax (Nouvelle-Écosse), Canada

4. Réseau atlantique pour la promotion de la santé communautaire, Université Dalhousie, Halifax (Nouvelle-Écosse), Canada

5. Faculté des professions de la santé, Université Dalhousie, Halifax (Nouvelle-Écosse), Canada

6. Service du médecin légiste de la Nouvelle-Écosse, Halifax (Nouvelle-Écosse), Canada

7. Régie régionale de la santé Capital, Halifax (Nouvelle-Écosse), Canada

8. Health LinQ, Université du Queensland, Queensland, Australie

Correspondance : Leslie Anne Campbell, bureau 230, Centre de recherche clinique, 5790, avenue University, Halifax (Nouvelle-Écosse) B3H 1V7; tél. : 902-473-7458; téléc. : 902-473-4546; courriel : leslie.anne.campbell@dal.ca. 
décès comme un suicide. En outre, certains médecins peuvent se montrer réticents à déclarer le suicide comme cause du décès en raison des préjugés ou des répercussions financières qui pèsent sur les membres de la famille ${ }^{5}$.

Lorsqu'on soupçonne un suicide, la nature du décès est déterminée selon un processus médico-légal pouvant reposer sur différents éléments de preuve : enquête sur la scène du décès, résultats d'autopsie, collecte d'antécédents médicaux et de renseignements circonstanciels, etc. $\mathrm{Au}$ Canada, les systèmes d'enquête sur les suicides varient selon les provinces et les territoires. Certains sont dotés d'un système de médecins légistes et d'autres d'un système de coroners ${ }^{6}$. Les médecins légistes sont des médecins, tandis que les coroners peuvent avoir une formation professionnelle dans le domaine du droit, des enquêtes ou de la médecine. En 1960, la Fatality Inquiries Act a instauré le Service du médecin légiste de la Nouvelle-Écosse (SMLNE); cette loi a été modifiée en 1989 afin d'établir le poste de médecin légiste en chef provincial $(\mathrm{CME})^{6}$. Dans sa version actuelle, le système du médecin légiste provincial fonctionne à partir d'un bureau central à Halifax, en Nouvelle-Écosse.

Le SMLNE est chargé d'enquêter sur tous les cas de mort violente, de mort non naturelle, de mort attribuable à la négligence coupable ainsi que sur les cas de décès inattendu ou inexpliqué survenus dans la province ${ }^{6}$, ce qui inclut tous les décès par suicide. Le rôle premier du SMLNE est d'identifier la personne décédée, d'établir la date, l'heure, le lieu et la cause du décès et, dans le cas d'un suicide manifeste, de déterminer l'intention. Ces fonctions sont décrites en détail dans la Fatality Investigations Act de la NouvelleÉcosse ${ }^{7}$. La portée de chaque enquête varie selon les circonstances du décès, mais le but est toujours de déterminer l'intention.

De toute évidence, il est nécessaire de classer avec précision le décès comme un suicide pour identifier les facteurs pouvant servir de cibles dans le cadre de stratégies d'intervention et de prévention. Toutefois, l'absence de critères normalisés de classification des suicides et la difficulté d'appliquer uniformément ces critères peuvent mener à une classification inexacte ${ }^{4,8,9}$.

Malgré ces contraintes, les dossiers des médecins légistes constituent des sources importantes de renseignements qui peuvent nous aider à mieux comprendre l'ampleur du suicide dans une population ainsi que les facteurs sociodémographiques et autres associés à ce phénomène $\mathrm{e}^{9-14}$. Des dossiers semblables se sont avérés utiles pour la recherche et la surveillance dans d'autres territoires, notamment ailleurs au Canada, en Angleterre et aux ÉtatsUnis $^{11,13,15-20}$. Toutefois, les renseignements recueillis par le SMLNE jusqu'à maintenant n’ont pas été utilisés pour la surveillance, mais seulement occasionnellement pour la recherche ${ }^{21}$.

Les recherches font ressortir plusieurs facteurs de risque individuels associés au suicide : de nombreuses personnes décédées ont des antécédents de problèmes de santé mentale ou de toxicomanie $e^{22,23,24}$, et les hommes et les garçons semblent présenter un risque élevé, souvent par l'utilisation de méthodes plus fatales s,15,20,25,26 $^{\text {. Parmi }}$ les autres facteurs de risque signalés, mentionnons un âge avancé ${ }^{26}$, le fait de vivre en milieu rural ${ }^{18}$, la possession d'une arme à feu à la maison ${ }^{27}$, l'isolement social ${ }^{25}$, un statut socioéconomique faible ${ }^{18,26}$, la douleur chronique, les maladies terminales ou les incapacités permanentes ${ }^{28}$ ou encore le fait d'être une victime ou un auteur de violence conjugale ${ }^{20}$.

Les enquêtes du SMLNE sont l'occasion de recueillir davantage de renseignements détaillés, notamment sur les facteurs de risque connus. De plus, le système du médecin légiste et les autres systèmes d'enquête sur les décès ont des mandats géographiques précis qui génèrent des sources de données populationnelles. Les actes de décès et les registres des traumatismes contiennent des renseignements incomplets sur les décès attribuables au suicide et, pour cette raison, ne peuvent à eux seuls orienter les politiques de prévention ou la recherche épidémiologique.

L'objectif de notre recherche consistait à examiner la teneur des renseignements recueillis par le SMLNE concernant les cas de suicide pour 1) déterminer les types et les sources de renseignements accessibles qui pourraient être utiles pour la recherche en prévention du suicide et 2) établir un " profil » des suicides afin de mettre en évidence les renseignements qui pourraient servir dans le cadre d'un système de surveillance continue. En ce qui concerne le dernier objectif, nous avons lié chacun des cas de suicide à des données sur les services de santé tirées des bases de données administratives de la province afin de dresser un profil de l'utilisation des services de santé au cours de l'année précédant le décès.

Le Comité d'examen éthique de l'Université Dalhousie et le ministère de la Justice de la Nouvelle-Écosse ont évalué et approuvé les travaux de recherche avant la collecte des données.

\section{Méthodologie}

Dans le cadre de l'étude, nous avons employé des méthodes mixtes : la composante qualitative, servant à évaluer les types et les sources de renseignements accessibles dans les dossiers, et la composante quantitative, permettant de fournir un " profil » des suicides survenus en Nouvelle-Écosse. Pour chacune des composantes, nous avons extrait à la main les données des dossiers du SMLNE pour tous les décès attribuables au suicide sur une période d'un an, soit du $1^{\mathrm{er}}$ janvier au 31 décembre $2006(\mathrm{n}=108)$. Nous avons choisi une période d'un an afin d'obtenir suffisamment de données pour évaluer de façon efficace les types et les sources de renseignements contenus dans les dossiers et pour établir un profil de suicides; l'année 2006 était la période la plus récente pour laquelle tous les dossiers relatifs au suicide étaient " clos ", c'est-à-dire qu'aucun nouveau renseignement ne pouvait être ajouté au dossier.

\section{Composante qualitative : types et sources de renseignements}

En ce qui concerne la composante qualitative de l'étude, notre adjoint à la recherche a examiné chaque dossier de suicide afin de consigner l'existence de données socioéconomiques et d'autres renseignements connexes (p. ex. l'endroit où le corps a 
été découvert) et de vérifier d'autres types et sources de renseignements contenus dans les dossiers. Par exemple, les renseignements transmis par un membre de la famille concernant la vie sociale de la personne décédée étaient indiqués comme suit : " un membre de la famille fournit des renseignements d'ordre social », sans autre information sur son identité.

Les données concernant les types et les sources d'autres renseignements ont été extraites et consignées dans un fichier texte. Ce dernier a ensuite été importé dans le logiciel NVivo 7 (QSR International) en vue de la gestion et du tri de l'information selon la source et le type de renseignements. Nos principaux spécialistes en recherche qualitative (LJ, $\mathrm{RB})$ ont régulièrement assuré une rétroaction durant le processus d'extraction et de tri afin de vérifier que la liste de types et de sources de renseignements était saisie de manière exhaustive. Tout au long de l'extraction des données, des notes ont été rédigées et constamment mises à jour afin que soit notée toute modification aux définitions préliminaires des catégories de sources et de types de renseignements. Des modifications ont été apportées lorsqu'une définition préliminaire a été jugée incomplète.

Nous avons créé des catégories distinctes (p. ex. questions d'ordre juridique, questions d'ordre social) pour les différents types de renseignements, et nous avons défini les sources comme étant les personnes (p. ex. les médecins), les lieux (p. ex. les morgues) et les documents (p. ex. les dossiers médicaux) qui fournissaient les renseignements.

\section{Composante quantitative : profil des suicides}

La composante quantitative de l'étude consistait à créer une base de données anonymisée avec Access 2003 (Microsoft) à partir des renseignements extraits manuellement des dossiers du SMLNE. La base de données comprenait des renseignements accessibles recueillis par le service du médecin légiste sur les facteurs démographiques, personnels et sociaux ainsi que des facteurs liés à l'événement

TABLEAU 1

Types de renseignements tirés des dossiers du SMLNE sur les cas de suicide ${ }^{a}$

\begin{tabular}{ll}
\hline Fréquence des renseignements & Type de renseignement \\
\hline Courants & Renseignements tirés de l'autopsie \\
(présents dans $60 \%$ des dossiers ou plus) & Cause du décès \\
& Circonstances du décès \\
& Avis de décès - procédures \\
& Enregistrement du décès \\
& Scène du décès \\
& Renseignements médicaux \\
& Activités immédiates antérieures \\
& $\begin{array}{l}\text { Activités/réponses du médecin/de la police } \\
\text { Lieu et détails/comptes rendus de la découverte du corps }\end{array}$ \\
& Renseignements sociodémographiques \\
& Renseignements sociopersonnels \\
Moins courants & Incidents graves de la vie courante \\
(présents dans moins de $60 \%$ des dossiers) & Problèmes d'ordre juridique \\
& Demande de renseignements ou de dossiers précis \\
(p. ex. dossiers dentaires) & Plans ou tentatives de suicide \\
\hline
\end{tabular}

Abréviation : SMLNE, Service du médecin légiste de la Nouvelle-Écosse.

a Tous les cas de décès par suicide ont été extraits manuellement des dossiers du SMLNE pour la période du $1^{\text {er }}$ janvier au 31 décembre 2006.

(p. ex. cause du décès, éléments déclencheurs) et l'utilisation antérieure de services de santé. Les données ont été exportées de la base de données, puis importées dans un chiffrier électronique Excel 2003 (Microsoft). Elles ont ensuite fait l'objet d'une vérification à la recherche d'erreurs et des données en double avant d'être analysées à l'aide du logiciel Statistical Package for Social Sciences, version 12 (SPSS).

Dans la mesure du possible, ces données ont été couplées aux bases de données administratives provinciales en vue de déterminer l'utilisation des services de santé par la personne décédée (services de santé généraux et de santé mentale à l'hôpital ou en consultation externe) dans la dernière année de sa vie. Nous avons utilisé les ensembles de données suivants que possède l'Unité de recherche en santé des populations (URSP) du Département de santé communautaire et d'épidémiologie de l’Université Dalhousie : la Base de données sur les congés des patients de l’Institut canadien d'information sur la santé (ICIS), qui contient les codes de diagnostic et les dates d'admission à l'hôpital et de congé des patients, les demandes de règlement des médecins et le Système d'information sur la santé mentale des patients externes.
Le couplage avec les bases de données a été rendu possible grâce aux numéros de cartes-santé encodés selon un processus approuvé par le ministère de la Justice, le Comité d'éthique de l'Université Dalhousie et le Comité d'accès aux données de l'URSP.

Nous avons calculé les données statistiques descriptives quantitatives (taux, pourcentages) pour chacun des facteurs démographiques déclarés dans les dossiers du SMLNE et l'utilisation antérieure de services de santé en Nouvelle-Écosse sur une période d'un an. La saisie incomplète de données nous a empêchés de procéder à une analyse des facteurs de risque connus.

Comme il fallait supprimer les petites cellules de données afin de préserver l'anonymat des personnes décédées, nous avons classé les consultations en santé uniquement en fonction des catégories "santé mentale » ou " autre que santé mentale », et les avons déclarées pour l'année et le mois ayant précédé le suicide. Malgré ce niveau de regroupement relativement élevé, nous avons été incapables de déterminer des types précis de soins de santé utilisés (c.-à-d. patients hospitalisés ou patients suivis en externe, santé mentale ou autre que santé mentale) au cours 
de la semaine précédant le suicide en raison du faible nombre de données. Il n’a pas été nécessaire d'effectuer un calcul de la taille de l'échantillon ou de la puissance, puisque le projet consistait à déclarer tous les cas de suicide en Nouvelle-Écosse pour la période donnée, et aucune hypothèse précise n’avait à être vérifiée.

\section{Résultats}

\section{Composante qualitative :}

types et sources de renseignements

En 2006, en Nouvelle-Écosse, sur une population de 913462 habitants, 108 décès étaient attribuables à un suicide ${ }^{29}$. Le SMLNE a mené une enquête sur chacun de ces décès et chaque cas a donc été consigné dans un dossier. Chacun des 108 dossiers présentait la même structure de base : des renseignements sur toutes les communications et tous les processus liés à l'événement, à partir du moment où l'a fait appel au médecin légiste jusqu'à la fermeture du dossier, ainsi que le rapport officiel du médecin légiste en chef. Toutefois, les détails présentés variaient énormément d'un dossier à l'autre.

Tous les dossiers contenaient des renseignements sur l'âge, le sexe, l'adresse de résidence, l'endroit où le corps avait été découvert et la cause du décès. D’autres renseignements sociodémographiques et connexes, par exemple, l'état matrimonial et la situation d'emploi, étaient plus ou moins enregistrés et pas toujours de façon cohérente. À titre d'exemple, dans une section du dossier, il pouvait être indiqué que la personne décédée était mariée, mais dans une autre section, qu'elle était séparée.

Nous avons distingué 16 types de renseignements (p. ex. données d'autopsie, scène du décès) à partir des 108 dossiers (tableau 1). Nous avons estimé que les renseignements étaient courants s'ils étaient présents dans $60 \%$ des dossiers ou davantage, et moins courants s'ils étaient présents dans moins de $60 \%$ des dossiers.

Nous avons également trouvé et classé 10 sources de renseignements. Cinq parmi elles ont été classées comme étant des sources courantes de renseignements, parce que la totalité ou la grande majorité (c.-à-d. $60 \%$ ou plus) des dossiers contenaient ces renseignements : famille/amis, dossiers médicaux, médecins légistes/ enquêteurs, médecins (y compris les médecins militaires) et police (y compris la police militaire). Moins de $60 \%$ des dossiers contenaient des renseignements issus d'autres sources. Il s'agissait surtout de données provenant de consultants (p. ex. neuropathologistes), de répondants médicaux d'urgence, de salons funéraires, de morgues et de banques de tissus.

\section{Composante quantitative}

La base de données quantitative dérivée comprenait les renseignements disponibles tirés des 108 dossiers, soit les données démographiques, le lieu du suicide, l'intention déclarée, la cause du décès, le recours antérieur à des soins de santé, les tentatives de suicide antérieures, les diagnostics médicaux et psychiatriques et les circonstances déclenchantes chez la personne décédée. Les données personnelles de base (âge, sexe, adresse, cause et lieu du décès) étaient consignées uniformément dans les dossiers.

À partir de cette base de données, nous avons déterminé que l'âge moyen des personnes décédées était de 44,7 ans (écarttype [ÉT] $\pm 13,3$ ans) et que celles dans la quarantaine constituaient le tiers des cas $(n=36)$ (tableau 2). Le ratio femmeshommes était de $1: 5$, avec 18 (16,7 \%) femmes et $90(83,3 \%)$ hommes. Un peu plus de la moitié des personnes composant l'échantillon vivaient dans des régions rurales (c'est-à-dire dans des régions situées à l'extérieur de la municipalité régionale de Halifax), ce qui est similaire à la moyenne de l'ensemble de la population de la Nouvelle-Écosse, qui était de $59 \%$ en $2006^{29}$. Les causes de décès les plus fréquentes étaient la pendaison $(38,9 \%)$, l'auto-intoxication $(24,1 \%)$ et les blessures par arme à feu $(19,4 \%)$. Les lieux les plus courants étaient le domicile (56,5\%) ou un endroit public comme un pont, un parc, un boisé ou une plage $(27,8 \%)$.

En ce qui concerne la cause du décès, les différences selon le sexe étaient évidentes : les formes les plus courantes de suicide chez les femmes et les jeunes filles étaient l'auto-intoxication $(38,9 \%)$ et la pendaison $(33,3 \%)$; chez les hommes, c'était la pendaison (40\%), les blessures par arme à feu (23,3 \%) et l'auto-intoxication $(21,1 \%)$. Toutes les personnes décédées à la suite d'une blessure par arme à feu étaient de sexe masculin, et les deux tiers de ces décès étaient causés par une décharge d'une arme d'épaule (carabine ou fusil de chasse). Même si les circonstances déclenchantes n'ont pas toutes été rapportées dans les dossiers du SMLNE, les 18 cas de suicide où une séparation récente avait été notée concernaient des hommes.

Mis à part les données personnelles de base, les dossiers ne contenaient pas tous les mêmes renseignements. Par exemple, même si l'âge et le sexe des personnes décédées figuraient dans tous les dossiers, il n'y avait aucune mention de la situation d'emploi dans 43,5\% de ceux-ci. La plupart des dossiers (69 \%) ne contenaient pas d'information sur des tentatives de suicide antérieures connues et dans $28 \%$ d'entre eux, il n'y avait aucun renseignement sur un quelconque diagnostic psychiatrique. Dans le cas de décès par pendaison, il manquait des données dans $98 \%$ des dossiers sur la source du lien, le point et le degré de suspension. De même, dans le cas de décès par arme à feu, $90 \%$ des dossiers ne contenaient aucune information sur la propriété, l'état du permis et l'entreposage de l'arme à feu.

Nous avons enrichi les données sur l'utilisation des services de santé en couplant nos bases de données dérivées avec les bases de données administratives provinciales sur les services de santé à l'aide des numéros de cartes-santé encodés. Parmi les personnes décédées dont il était possible d'extraire le numéro de carte-santé ( $n=101)$, la plupart (74 \%) avaient reçu des services de santé (à l'hôpital ou en consultation externe) au cours de l'année précédant le suicide; plus de la moitié (55\%) de ces personnes avaient été suivies en tant que patients externes et près du quart (23\%) avaient été hospitalisées pour des raisons de santé 
mentale dans l'année précédant le suicide; $10 \%$ des personnes avaient été admises à l'hôpital pour des raisons de santé mentale au cours du mois précédant le suicide; $16 \%$ avaient eu recours d'une manière ou d'une autre au système de soins de santé dans la semaine ayant précédé le suicide; enfin, $9 \%$ avaient été suivis en consultation externe par un omnipraticien ou un psychiatre pour des raisons de santé mentale dans la semaine précédant le suicide

\section{Analyse}

Les dossiers du SMLNE peuvent constituer une source précieuse de renseignements pour la surveillance et les efforts de prévention du suicide. Ils peuvent fournir des données plus détaillées que les bases de données administratives provinciales sur les services de santé, car ils contiennent des renseignements sur des circonstances déclenchantes telles qu'une séparation, des problèmes conjugaux, une perte d'emploi, des situations de violence (en tant qu'agresseur ou victime), des problèmes d'ordre juridique, des problèmes à l'école, au travail ou encore des problèmes financiers. Comme le SMLNE pratique une autopsie sur toutes les personnes décédées par suicide ou potentiellement par suicide, ses dossiers comprennent nécessairement une source beaucoup plus complète de problèmes médicaux concomitants que toute autre base de données.

Notre examen des dossiers du SMLNE a révélé que certains renseignements sociodémographiques (c.-à-d. l'âge, l'adresse, le sexe, l'état matrimonial) étaient consignés dans tous les dossiers, même si l'état matrimonial n'était pas toujours inscrit de façon régulière. Il manquait parfois d'autres renseignements (p. ex. la situation d'emploi) qui seraient utiles à des fins de surveillance et de recherche en prévention. Nos résultats correspondent à ceux d'une étude menée en 2005 sur les dossiers de coroners en Angleterre, dans laquelle des caractéristiques démographiques comme le sexe, l'âge et l'état matrimonial étaient généralement bien inscrites, mais où il manquait des renseignements relatifs à l'emploi dans plus du tiers des cas, ce qui empêchait d'établir une classification socioéconomique solide ${ }^{13}$.
L'enregistrement relativement constant des renseignements démographiques de base nous a permis de dresser un profil général de personnes qui sont décédées par suicide en Nouvelle-Écosse sur une période d'un an. Les taux supérieurs que nous avons constatés chez les hommes, les garçons et les personnes dans la quarantaine correspondaient aux modèles observés dans

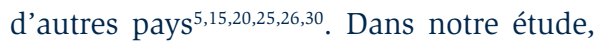
comme dans d'autres, les hommes et les garçons étaient plus nombreux à employer des méthodes de suicide vraiment fatales, augmentant ainsi la probabilité que le décès soit classé comme un suicide ${ }^{15,20,25}$. En raison du manque d'uniformité dans la collecte de renseignements concernant d'autres facteurs de risque, nous n'avons pu procéder à des analyses plus raffinées.

Les renseignements sur les facteurs de risque connus, comme les problèmes de santé, peuvent orienter la surveillance et les efforts de prévention. En particulier, les données concernant les maladies mentales (p. ex. les troubles de la personnalité ou les épisodes de dépression majeure), la consommation d'alcool et de drogues ou les multiples problèmes de santé physique chroniques sont utiles puisque ces problèmes de santé exposent à un risque plus élevé de suicide ${ }^{22,31}$. Toutefois, ces renseignements ont été inclus de façon variable dans les dossiers que nous avons examinés. Nous avons pu, dans une certaine mesure, surmonter cette lacune en établissant un lien avec les dossiers médicaux provenant des bases de données administratives provinciales lorsque nous avions accès aux numéros encodés valides des cartes-santé des personnes décédées (101 dossiers sur 108). Néanmoins, une bonne partie des renseignements médicaux ne figuraient pas systématiquement dans les dossiers du SMLNE.

Les données sur les armes à feu peuvent être particulièrement utiles pour guider les politiques publiques. Notre étude montre que la plupart des dossiers ne contiennent cependant aucun renseignement sur la propriété, l'état du permis et l'entreposage. Dans les cas de décès par pendaison, l'information concernant le lien est tout aussi rare. Cette information est peut-être moins pertinente pour l'élaboration de politiques puisque le matériel nécessaire pour se pendre est largement accessible au public. De tels renseignements seraient néanmoins plus pertinents en milieu institutionnel.

D'autres facteurs et conditions socioéconomiques, comme le fait de recevoir des prestations d'invalidité, avoir un faible revenu ou un faible niveau de scolarité, vivre une perte importante (p. ex. rupture d'une relation personnelle ou perte d'emploi), posséder un casier judiciaire (et les craintes liées à une arrestation ou à un emprisonnement) et connaître un isolement social constituent également d'importants facteurs de risque de suicide potentiels $^{32-34}$. Ces renseignements ne sont pas toujours non plus consignés dans les dossiers du SMLNE. Ce problème n'est pas propre à la Nouvelle-Écosse ni au Canada : de telles limites ont également été observées par des chercheurs chargés d'examiner les rapports de coroners ou de médecins légistes ailleurs ${ }^{9,13}$.

$\mathrm{Au}$ moins quatre raisons principales semblent expliquer les écarts entre les dossiers de même que les incohérences dans les dossiers : 1) les renseignements sont recueillis par des personnes différentes (c.-à-d. divers enquêteurs, policiers, etc.) susceptibles de consigner l'information à des degrés divers; 2) les renseignements proviennent de différentes sources (p. ex. famille, amis ou médecins) susceptibles de connaître la personne décédée sous différents aspects et à divers degrés, ou qui peuvent interpréter différemment les questions de l'enquêteur; 3) les dossiers médicaux des personnes décédées ne sont pas toujours exigés, obtenus ou consignés de manière uniforme et 4) il n'y aucune méthode d'entrevue structurée en place pour recueillir les renseignements médicaux et sociaux fournis en particulier par la famille et les amis. La collecte de renseignements provenant des membres de la famille ou d'amis peut être également compliquée par la réticence de ces derniers à faire état de déclarations d'intention pour des raisons financières (p. ex. les assurances) ou à cause des préjugés associés au suicide.

Toutes ces explications convergent vers un principe fondamental : la collecte de 
TABLEAU 2

Profils des cas de suicide tirés des dossiers du SMLNE pour la période du $1^{\mathrm{er}}$ janvier au 31 décembre $2006(\mathrm{~N}=108)^{\mathrm{a}}$

\begin{tabular}{|c|c|c|}
\hline \multirow{2}{*}{$\begin{array}{l}\text { Caractéristiques } \\
\text { Âge }\end{array}$} & \multicolumn{2}{|c|}{$\begin{array}{c}\text { Nombre de cas } \\
\text { n (\%) }\end{array}$} \\
\hline & & \\
\hline $\begin{array}{l}\text { Moins de } 30 \text { ans } \\
30-39 \text { ans } \\
40-49 \text { ans } \\
50-59 \text { ans } \\
60-69 \text { ans } \\
70 \text { ans et plus }\end{array}$ & $\begin{array}{r}15 \\
23 \\
36 \\
21 \\
7 \\
6\end{array}$ & $\begin{array}{r}(13,9) \\
(21,3) \\
(33,3) \\
(19,4) \\
(6,5) \\
(5,6)\end{array}$ \\
\hline \multicolumn{3}{|l|}{ Sexe } \\
\hline $\begin{array}{l}\text { Homme } \\
\text { Femme }\end{array}$ & $\begin{array}{l}90 \\
18 \\
\end{array}$ & $\begin{array}{r}(83,3) \\
(1,7) \\
\end{array}$ \\
\hline $\begin{array}{l}\text { Lieu de résidence } \\
\text { Milieu urbain } \\
\text { Milieu rural }\end{array}$ & $\begin{array}{l}52 \\
56 \\
\end{array}$ & $\begin{array}{l}(48,1) \\
(51,9) \\
\end{array}$ \\
\hline $\begin{array}{l}\text { Cause du décès } \\
\text { Pendaison } \\
\text { Auto-intoxication } \\
\text { Blessure par } \\
\text { arme à feu } \\
\text { Noyade } \\
\text { Lésion par force } \\
\text { contondante } \\
\text { Autre }\end{array}$ & $\begin{array}{r}42 \\
26 \\
21 \\
\\
6 \\
5\end{array}$ & $\begin{array}{r}(38,9) \\
(24,1) \\
(19,4) \\
(5,6) \\
(4,6) \\
(7,4)\end{array}$ \\
\hline \multicolumn{3}{|l|}{ Lieu du décès } \\
\hline $\begin{array}{l}\text { Domicile } \\
\text { Endroit public } \\
\text { (pont, boisé) }\end{array}$ & $\begin{array}{l}61 \\
30\end{array}$ & $\begin{array}{l}(56,5) \\
(27,8)\end{array}$ \\
\hline $\begin{array}{l}\text { Véhicule } \\
\text { Propriété de la } \\
\text { famille ou d'un ami }\end{array}$ & $\begin{array}{l}6 \\
6\end{array}$ & $\begin{array}{l}(5,6) \\
(5,6)\end{array}$ \\
\hline Autre & 5 & $(4,6)$ \\
\hline
\end{tabular}

Abréviation : SMLNE, Service du médecin légiste de la Nouvelle-Écosse.

a Tous les cas de décès par suicide ont été extraits manuellement des dossiers du SMLNE pour la période du $1^{\text {er }}$ janvier au 31 décembre 2006.

données par un coroner ou un médecin légiste s'effectue dans un contexte d'enquête et non de recherche. En ce sens, le but est de déterminer la cause et les circonstances du décès d'une personne et, dans le cas d'un suicide, de déterminer l'intention, et non de recueillir des données normalisées. Par contre, la collecte systématique de données permettrait de constituer une source précieuse de données utiles pour la surveillance en population et les activités de recherche en prévention.

La pertinence de ces renseignements ne se limite pas à la Nouvelle-Écosse. Statistique Canada a mis sur pied la Base canadienne de données des coroners et des médecins légistes (BCDCML). Celle-ci conservera les données sur les décès déclarés aux coroners et aux médecins légistes afin de faciliter l'identification des sources de danger pour la sécurité connues ou nouvelles et d'en définir les caractéristiques, contribuant ainsi à la prévention de décès évitables chez les Canadiens ${ }^{35}$. La capacité de la BCDCML d'atteindre cet objectif dépendra de la qualité et de l'intégralité des données.

Afin de fournir une base de donnée néoécossaise représentative et complète, nous recommandons que les champs de collecte soient définis et alimentés de façon constante durant les enquêtes des médecins légistes. Même si les répercussions opérationnelles de ce changement sont minimes, celui-ci marque une rupture sur le plan conceptuel par rapport à la façon dont le SMLNE est décrit actuellement dans la loi.

\section{Limites}

Composante qualitative. Comme les données ont été recueillies sur une période d'un an, il se peut que d'autres types et sources de renseignements aient été consignés dans les dossiers en dehors de cette période. Toutefois, beaucoup de types et de sources de données trouvées dans notre étude étaient les mêmes d'un dossier à l'autre, ce qui donne à penser que nous avons été en mesure de recueillir la majorité, sinon la totalité, des types et sources de renseignements.

Composante quantitative. Nous avions prévu de déterminer si certains types de renseignements sur le suicide (p. ex. des données sur des problèmes de santé mentale) pouvaient être recueillis de façon systématique en lien avec des caractéristiques démographiques (p. ex. groupe d'âge ou sexe). Lors de la collecte et de l'analyse des données, nous avons découvert que peu de questions structurées étaient posées de façon constante sur chaque suicide, ce qui s'est traduit par une saisie incomplète des données rendant impossible la réalisation de ce type d'analyse. Dans le cas de l'utilisation de services de santé, nous avons pu surmonter cet écueil en établissant un lien avec des bases de données administratives, mais nous étions par ailleurs incapables d'établir des associations entre des variables identifiées comme des facteurs de risque de suicide (p. ex. problèmes de santé mentale).

Les petites cellules de données constituaient également une limite. Nous avons supprimé les cellules inférieures à 5 pour éviter l'identification des personnes par inadvertance. Des travaux ultérieurs pourraient intégrer la préparation d'une cohorte portant sur un intervalle de temps plus long.

\section{Conclusion}

Jusqu'à maintenant, les données recueillies par le SMLNE dans le cadre de ses enquêtes n'ont pas été analysées ni utilisées à des fins de recherche en surveillance ou en prévention continue. Notre étude a révélé qu'une bonne partie des renseignements recueillis par les médecins légistes en Nouvelle-Écosse sont variables et qu'ils ne peuvent ainsi complètement servir à élaborer un système de surveillance rigoureux et représentatif à l'échelle provinciale qui inclut un certain nombre de facteurs de risque de suicide. L'utilisation des données provenant des médecins légistes pour la surveillance et la recherche en prévention du suicide semble poser deux problèmes importants : 1) des incohérences d'un dossier à l'autre concernant certains renseignements sociodémographiques recueillis et consignés, de même que des incohérences dans un même dossier et 2) des écarts importants d'un dossier à l'autre quant à la quantité de renseignements sociaux, médicaux et autre fournis ou consignés.

L’utilisation de données recueillies de façon systématique offre des moyens réalistes de surveillance. Les dossiers du SMLNE peuvent fournir des renseignements sur tous les décès réputés être attribuables au suicide. L'utilisation d'un outil normalisé d'entrevue ou d'un outil de collecte de données dans le cadre d'enquêtes assurerait l'intégralité des données. Il pourrait s'agir de questions fermées qui serviraient à alimenter une base de données; toutefois, nous sommes conscients que le caractère unique de chaque enquête empêche la mise en place d'un seul ensemble uniforme de questions fermées. L'ensemble 
de données exhaustives qui en résulte peut nous aider à mieux comprendre le suicide à l'échelle de la population, notamment l'emploi de méthodes communes et les facteurs sociodémographiques associés, de même qu'à définir les possibi-lités d'intervention. La reconstruction du SMLNE en vue de répondre à ce besoin important en santé publique exigera probablement des modifications législatives.

\section{Remerciements}

Les auteurs tiennent à remercier le ministère de la Justice de la NouvelleÉcosse de leur avoir permis d'accéder aux dossiers du Service du médecin légiste de la Nouvelle-Écosse (SMLNE). Les données administratives ont été fournies par l'Unité de recherche en santé des populations (URSP) du Département de santé communautaire et d'épidémiologie de l'Université Dalhousie. La province de la Nouvelle-Écosse fournit à l'URSP les données relatives à la facturation médicale et aux congés des patients des hôpitaux qui conviennent aux travaux de recherche. Cette étude a été financée par le ministère de la Promotion et de la Protection de la santé de la Nouvelle-Écosse. Les avis, résultats et conclusions de l'étude n'engagent que les auteurs et sont indépendants des sources de financement et de données. Ils ne reflètent la position ni du ministère de la Santé et du Bien-être ni du ministère de la Justice de la Nouvelle-Écosse et ne devraient pas être perçus comme tels.

\section{Références}

1. Peter Nestman; Population Health Research Unit. Suicide and attempted suicide in Nova Scotia (1995-2004): a report [Internet]. Halifax (NS): Department of Health Promotion and Protection; février 2009 [consulté le 10 janvier 2011]. PDF (1,09 Mo) téléchargeable à partir du lien : http://www.gov.ns.ca/hpp/publications/ Suicide_Report.pdf.
2. Statistique Canada. CANSIM Tableau 1024309. Mortalité et années potentielles de vie perdues, selon certaines causes de décès et le sexe, moyenne de trois ans, Canada, provinces, territoires, régions sociosanitaires et groupes de régions homologues, occasionnel [Internet]. Ottawa (Ontario) : Statistique Canada; [consultation le 18 avril 2011]. Consultable en ligne à partir de la page : http://www5.statcan .gc.ca/cansim/pick-choisir?lang=fra\&search TypeByValue $=1$ \&id $=1024309$

3. Donaldson AE, Larsen Y, Fullerton-Gleason L, Olson LM. Classifying undetermined poisoning deaths. Inj Prev. 2006;12:338-43.

4. Parai J, Kreiger N, Tomlinson G, Adlaf E. The validity of the certification of manner of death by Ontario coroners. Ann Epidemiol. 2006;16:805-11.

5. Santé Canada. Rapport sur les maladies mentales au Canada. Ottawa (Ontario) : Santé Canada; octobre 2002 [cité le 13 juillet 2010]. PDF (2,68 Mo) téléchargeable à partir du lien : http://www.phac-aspc.gc.ca/ publicat/miic-mmac/pdf/men_ill_f.pdf.

6. Government of Nova Scotia. Office of the Chief Medical Examiner. Halifax (NS): Government of Nova Scotia; [mis à jour le 4 avril 2009; consultation le 13 juillet 2010]. Consultable en ligne à la page : http://www .gov.ns.ca/just/CME.asp.

7. Government of Nova Scotia. Fatality investigations act, 2001. Halifax (NS): Government of Nova Scotia; 2002 [consultation le 13 juillet 2010]. Consultable en ligne à la page : http://nslegislature.ca /legc/statutes/fatalinv.htm.

8. Moscicki EK. North American perspectives: epidemiology of suicide. Int Psychogeriatr. 1995;7:137-48.

9. Parrish G. Assessing and improving the quality of data from medical examiners and coroners [Internet]. Atlanta (GA): Centres for Disease Control and Prevention; 1995 [consultation le 13 juillet 2010]. PDF (560 Ko) téléchargeable à partir du lien : http://www .cdc.gov/nchs/data/ice/ice95v1/C25.pdf.
10. Isaacs S, Keogh S, Menard C, Hockin J. Le suicide dans les Territoires du NordOuest : un examen descriptif. Mal. chron. Can. 1998;19:167-171.

11. Poulin C, Stein J, Butt J. Surveillance des décès par surdose à l'aide des données des médecins légistes. Mal. chron. Can. 1998;19:194-199.

12. Snowdon J, Baume P. A study of suicides of older people in Sydney. Int J Geriatr Psychiatry. 2002;17:261-9.

13. Bennewith O, Hawton K, Simkin S, Sutton L, Kapur N, Turnbull P, et al. The usefulness of coroners' data on suicides for providing information relevant to prevention. Suicide Life Threat Behav. 2005;35:607-14.

14. Comstock RD, Mallonee S, Jordan F. A comparison of two surveillance systems for deaths related to violent injury. Inj Prev. 2005;111:58-63.

15. Shields LB, Hunsaker DM, Hunsaker JC 3rd. Suicide: a ten-year retrospective review of Kentucky medical examiner cases. J Forensic Sci. 2005 May;50(3):613-7.

16. Juurlink DN, Mamdani MM, Kopp A, Redelmeier DA. The risk of suicide with selective serotonin reuptake inhibitors in the elderly. Am J Psychiatry. 2006;163:813-21.

17. Sutton L, Hawton K, Simkin S, Turnbull P, Kapur N, Bennewith O, et al. Gunshot suicides in England--a multicentre study based on coroners' records. Soc Psychiatry Psychiatr Epidemiol. 2005 Apr;40:324-8.

18. Hempstead K. The geography of self-injury: spatial patterns in attempted and completed suicide. Soc Sci Med. 2006;62:3186-96.

19. Paulozzi LJ, Mercy J, Frazier L Jr, Annest JL; Centers for Disease Control and Prevention. CDC's National Violent Death Reporting System: background and methodology. Inj Prev. 2004;10:47-52.

20. Sanford C, Marshall SW, Martin SL, CoyneBeasley T, Waller AE, Cook PJ, et al. Deaths from violence in North Carolina, 2004: how deaths differ in females and males. Inj Prev. 2006 Dec;12 Suppl 2:ii10-ii16. 
21. Croix-Rouge canadienne. Les faits sur la noyade au Canada : édition 2005 [Internet]. Ottawa (Ont.) : Croix-Rouge canadienne; 2005 [consultation le 20 octobre 2010]. PDF (1,17 Mo) téléchargeable à partir du lien : http://www.croixrouge.ca/cmslib/general /drowningreport2005_fr.pdf.

22. Cheng AT, Chen TH, Chen CC, Jenkins R. Psychosocial and psychiatric risk factors for suicide. Case-control psychological autopsy study. Br J Psychiatry. 2000;177:360-5.

23. Séguin M, Lesage A, Turecki G, Daigle F, Guy A. Projet de recherche sur les décès par suicide au Nouveau-Brunswick entre avril 2002 et mai 2003 [Internet]. Montréal (Québec) : Institut universitaire en santé mentale Douglas; avril 2005 [consultation le 13 juillet 2010]. PDF (686 Ko) téléchargeable à partir du lien : http://www.gnb .ca/0055/pdf/3182-f.pdf.

24. Garlow SJ. Age, gender, and ethnicity differences in patterns of cocaine and ethanol use preceding suicide. Am J Psychiatry. 2002 Apr;159:615-9.

25. Langlois S, Morrison P. Suicides et tentatives de suicide. Rapports sur la santé. 2002;13(2):9-26.

26. Paris J. Predicting and preventing suicide: do we know enough to do either? Harv Rev Psychiatry. 2006;14:233-40.

27. Shields LB, Hunsaker DM, Hunsaker JC 3rd. Adolescent and young adult suicide: a 10-year retrospective review of Kentucky Medical Examiner cases. J Forensic Sci. 2006 Jul;51(4):874-9.

28. Rowe JL, Bruce ML, Conwell Y. Correlates of suicide among home health care utilizers who died by suicide and community controls. Suicide Life Threat Behav. 2006 Feb;36(1):65-75.
29. Statistique Canada. 2006 Profils des communautés : Halifax, Nouvelle-Écosse (Code 1209034) (tableau) [Internet]. Ottawa (Ontario): Statistique Canada; 2007 [consultation le 6 janvier 2011]. [Statistique Canada, No 92-591-XWF au catalogue]. Consultable à partir de la page : http://www12.statcan.ca /census-recensement/2006/dp-pd/prof/92

$-591 /$ index.cfm? Lang $=\mathrm{F}$.

30. Hu G, Wilcox HC, Wissow L, Baker SP. Mid-life suicide: an increasing problem in U.S. whites, 1999-2005. Am J Prev Med. 2008;35:589-93.

31. Qin P, Agerbo E, Mortensen PB. Suicide risk in relation to socioeconomic, demographic, psychiatric, and familial factors: a national register-based study of all suicides in Denmark, 1981-1997. Am J Psychiatry. 2003;160:765-72.

32. Chan WS, Yip PS, Wong PW, Chen EY. Suicide and unemployment: what are the missing links? Arch Suicide Res. 2007;11:327-35.

33. Rush B, Koegl CJ. Prevalence and profile of people with co-occurring mental and substance use disorders within a comprehensive mental health system. Can J Psychiatry = Rev. can. psych. 2008;53:810-21.

34. Stack S, Wasserman I. Economic strain and suicide risk: a qualitative analysis. Suicide Life Threat Behav. 2007;37:103-12.

35. Statistique Canada. Base canadienne de données des coroners et des médecines légistes (BCDCML). Ottawa (Ontario): Statistique Canada; [modifié le 16 décembre 2009; consultation le 13 juillet 2010]. Consultable à partir de la page : http:// www.statcan.gc.ca/cgi-bin/imdb/p2SV_f .pl? Function $=$ getSurvey \&SDDS $=5125 \&$ lang $=e n \& d b=i m d b \& a d m=8 \& d i s=2$ 